\title{
Sunlight harvested by nanotubes
}

Rolling an atom-thick semiconductor layer into a nanoscale tube allows it to convert solar energy into electricity without the need for semiconductor junctions - prerequisite features of conventional solar cells. SEE LETTER P.349

\section{MING-MIN YANG \& MARIN ALEXE}

$\mathrm{F}$ or decades, the development of a cheap and efficient way to convert sunlight into electricity has been at the forefront of research, from the physical sciences to engineering. Usually, devices for harvesting solar energy, called solar cells, are made of semiconductors such as silicon. In these devices, electrical power is generated at the junction between two types of semiconductor material. However, the efficiency of junction-based solar cells has almost reached its theoretical limit, and it is therefore imperative to explore methods for converting sunlight into electricity that do not require semiconductor junctions. On page 349, Zhang et al. ${ }^{1}$ report a key advance in this direction. They demonstrate a junction-free solar cell that is produced by curling an atom-thick semiconductor layer into a nanoscale tube.

In a conventional solar cell, different chemical elements are added to two regions of a semiconductor in a process known as doping. Electrical transport occurs through negatively charged electrons in one region and through positively charged electron vacancies, called holes, in the other. An electric field is generated at the junction between these two regions. When sunlight is absorbed at this junction, electron-hole pairs are produced. The electrons and holes are then separated by the electric field, giving rise to an electric current (Fig. 1a). This conversion of solar energy into electricity is known as the photovoltaic effect.

Zhang and colleagues fabricated junctionfree solar cells using the semiconductor tungsten disulfide. Crystals of this material have a layered structure, and can be peeled off layer by layer in a similar way to graphite. The resulting atom-thick sheets can then be rolled by chemical methods into tubes that have diameters of about 100 nanometres. The authors made devices from three types of tungsten disulfide: a monolayer, a bilayer and a nanotube (see Fig. 1a of the paper ${ }^{1}$ ).

The authors found that, whereas the monolayer and bilayer devices generated a negligible electric current under illumination, the nanotube device exhibited a large photovoltaic effect. Given that these three types of solar cell have the same, uniform chemical identity, how does the nanotube device convert light into electricity
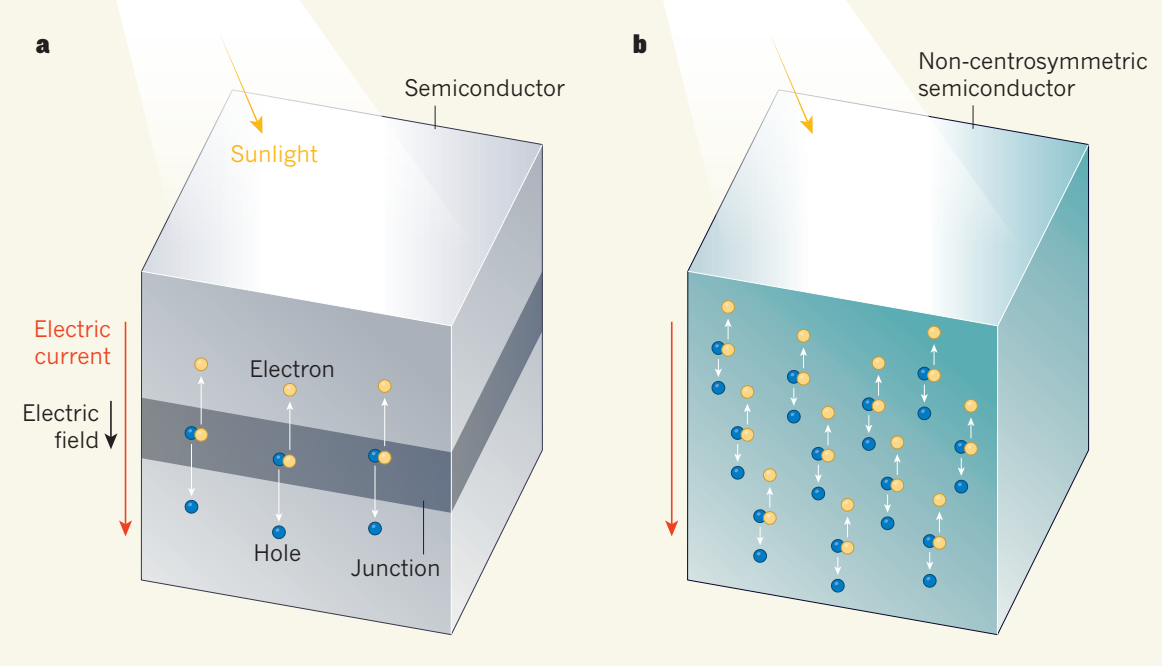

Figure 1 | Two types of solar cell. a, A conventional solar cell is made of a semiconductor such as silicon. Electrical transport occurs through electron vacancies called holes in one region (bottom), and through electrons in another region (top). An electric field is generated across the junction between these two regions. When this junction is illuminated by sunlight, electron-hole pairs are produced. The electrons and holes are then separated by the electric field, giving rise to an electric current. $\mathbf{b}$, Zhang et al. ${ }^{1}$ report a junction-free solar cell that is made of a non-centrosymmetric semiconductor - one whose structure lacks symmetry under a transformation known as spatial inversion. Under illumination, electron-hole pairs are produced and separated because of a phenomenon called the bulk photovoltaic effect, generating an electric current.

without the assistance of a junction? And why is this ability absent in the monolayer and bilayer devices? Zhang et al. point to a phenomenon called the bulk photovoltaic effect (BPVE), and attribute the diverse performance of the solar cells to their distinctive crystal symmetries. The BPVE can spontaneously generate a current in a uniform semiconductor, without the requirement for a junction (Fig. 1b).

The BPVE was first observed ${ }^{2}$ at Bell Labs in New Jersey in 1956, just two years after the invention of modern silicon solar cells ${ }^{3}$. The effect is restricted to non-centrosymmetric materials ${ }^{4}$, which are characterized by a lack of symmetry under spatial inversion (the combination of $180^{\circ}$ rotation and a reflection). The BPVE has two intriguing features: the lightgenerated current depends on the polarization of the incident light, and the associated voltage is larger than the material's bandgap (the energy required to excite conducting free electrons). However, the effect has a typically low conversion efficiency, and so has remained of academic rather than practical interest over the years.

To achieve a high BPVE efficiency, a material must have high light absorption and low internal symmetry. However, these two properties do not usually coexist in a given material. Semiconductors that absorb most incident sunlight generally have high symmetry, which diminishes or even prevents the BPVE. And common materials that have low symmetry, such as compounds called perovskite oxides, absorb little sunlight owing to their large bandgap. To circumvent this problem, tremendous effort has been devoted to enhancing light absorption in materials that have low symmetry, for example by using doping ${ }^{5}$. Meanwhile, it has been shown that the BPVE can be enabled in semiconductors when it is otherwise prohibited, by using mechanical fields to tailor the material's crystal symmetry ${ }^{6}$.

Zhang and colleagues' work calls attention to a hitherto-unexplored approach: shaping the semiconductors that have high light 
absorption into nanotubes. In the case of tungsten disulfide, the crystal symmetry of the nanotube is reduced with respect to that of the monolayer and bilayer, because of the tube's curved walls. The combination of excellent light absorption and low crystal symmetry means that the nanotube exhibits a substantial BPVE. The density of the electric current associated with the BPVE surpasses that of the materials that have inherently low symmetry, even though the conversion efficiency of the BPVE is still much lower than that of the junction-based photovoltaic effect in conventional solar cells.

The authors' results demonstrate the great potential of nanotubes in harvesting solar energy, and raise several technological challenges and scientific questions. From an applications perspective, it would be instructive to fabricate and characterize a solar cell that is made up of an array of semiconductor nanotubes, to check the feasibility of scaling up the approach. The direction of the current generated by the BPVE in each nanotube would be largely determined by the material's internal symmetry. Therefore, a uniform symmetry across the nanotube array would be needed to gather a collective current from the solar cell. In a worst-case scenario, if the currents generated in different nanotubes were in opposite directions, they would cancel each other out.

An important but unanswered question is whether the BPVE and the junction-based photovoltaic effect could cooperate in the same solar cell, to boost the overall efficiency. These two effects could harness solar energy in a successive manner. Nevertheless, despite the remaining challenges, Zhang and colleagues' work provides a possible route towards the design of highly efficient, unconventional solar cells. .

Ming-Min Yang and Marin Alexe are in the Department of Physics, University of Warwick, Coventry CV4 7AL, UK.

e-mails:mingmin.yang.1@warwick.ac.uk; m.alexe@warwick.ac.uk

1. Zhang, Y. J. et al. Nature 570, 349-353 (2019).

2. Chynoweth, A. G. Phys. Rev. 102, 705-714 (1956).

3. Chaplin, D. M., Fuller, C. S. \& Pearson, G. L. J. Appl. Phys. 25, 676-677 (1954).

4. Fridkin, V. M. Photoferroelectrics (Springer, 1979).

5. Grinberg, l. et al. Nature 503, 509-512 (2013).

6. Yang, M. M., Kim, D. J. \& Alexe, M. Science $\mathbf{3 6 0}$, 904-907 (2018).

\section{Sex chromosomes manipulate mate choice}

Female mate choice in some species selects for traits that are harmful to males. A hypothesis to explain how such mating preferences might evolve puts the spotlight on sex chromosomes. SEE LETTER P.376 that affect mating preferences have a range of effects on other traits. This is the most probable explanation for why females of some species have pre-existing preferences for traits that don't exist in males of their own species. For example, males of some species of swordtailed fishes have tails with greatly elongated lower fin rays. Females from a related species in which males do not have this 'sword' structure nevertheless have a preference for it, as assessed by their attraction to males of their own species that have had an artificial sword surgically attached to them ${ }^{3}$.

The second kind of hypothesis rests instead on indirect selection. Genetic variation in genes that affect mating preferences becomes correlated with genetic variation for other traits, and selection acting directly on the latter
A mong some of the most spectacular sights in nature are the bizarre mating displays of certain animals. These displays risk decreasing male survival because the bright colours and loud calls of the males might attract predators (Fig. 1). Yet despite this risk of harm, these traits have nevertheless evolved because only the males that can make the most exaggerated displays are chosen by females as mates. An enduring mystery in evolutionary biology is why females of these species have evolved such mating preferences. On page 376 , Muralidhar ${ }^{1}$ uses a population-genetics model to show how these types of preference can evolve if the versions of genes that contribute to the male mating display have beneficial effects when those versions of genes are present in females.

A variety of hypotheses for how female mating preferences evolve have been inspired by results from fieldwork, experiments and mathematical modelling. Most of these hypotheses fall into one of two main camps ${ }^{2}$. In the first, mating preferences evolve by direct selection - the genes that affect mating preferences are themselves direct targets of selection. For example, if some males offer superior parental care, female mating preferences for choosing those males will spread by natural selection. This is why females in monogamous species often prefer males that are not brightly coloured and that are therefore more likely to evade predators and survive to help rear offspring.

Direct selection can also act when genes

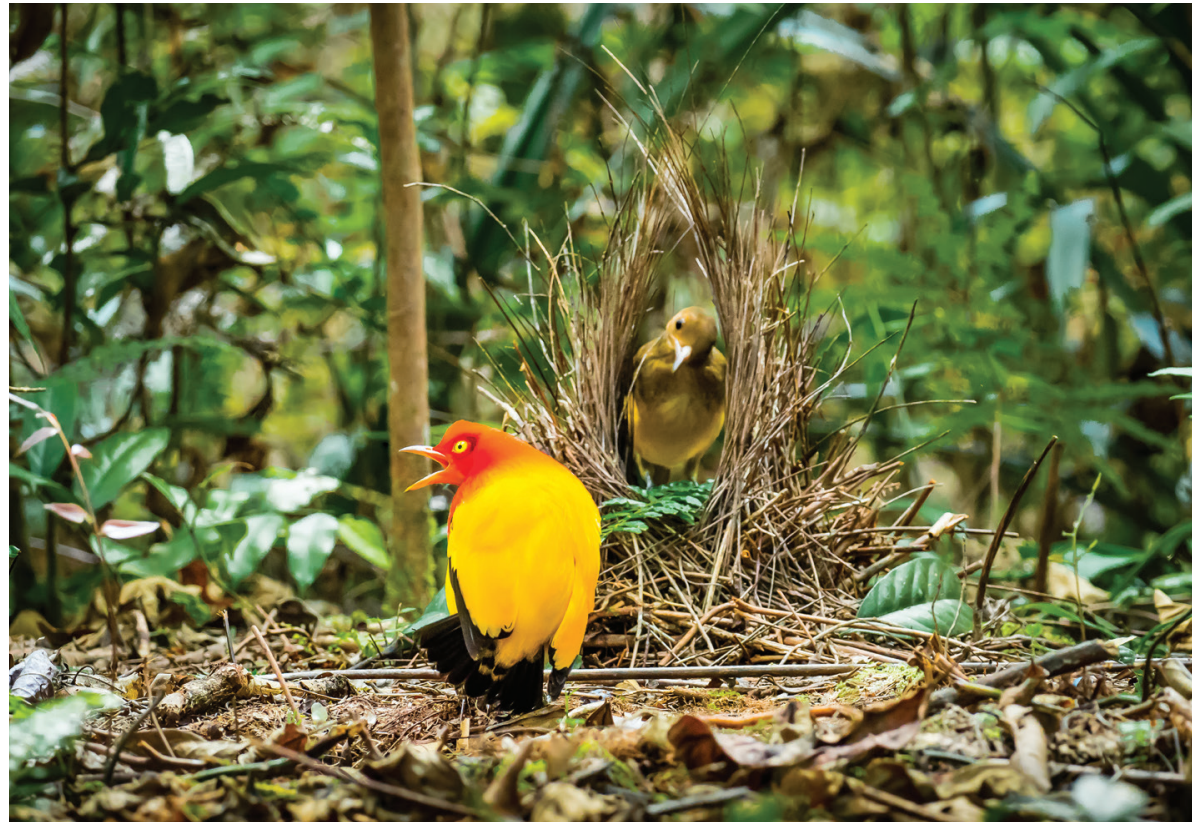

Figure 1 | A colourful mating display. A vibrantly coloured male flame bowerbird (Sericulus aureus) engages in a mating display as a female of the species looks on. The male's bright colours make the bird easy for predators to spot, raising the conundrum of how female mating preferences evolve for traits that are harmful to males. Muralidhar ${ }^{1}$ puts forward a hypothesis for how such mating preferences might arise. Whether this idea could explain the bowerbird case is unknown. 